\title{
Letter to the Editor on Type 1 diabetes onset triggered by COVID-19
}

\author{
Mentari Maratus Sholihah ${ }^{1} \cdot$ Taufik Ridwan Hadi Kusuma $^{1} \cdot$ Muhammad Ifham Hanif $^{2} \mathbb{D} \cdot$ Nurhasan Agung Prabowo $^{3}$
}

Received: 27 May 2021 / Accepted: 7 June 2021 / Published online: 28 June 2021

(c) Springer-Verlag Italia S.r.l., part of Springer Nature 2021

We read with great interest a published article in Acta Diabetologica 2020 entitled Type 1 diabetes onset triggered by COVID-19 [1]. This article has broad implications for readers, in which the literature has shown that around 0.6-46.2\% of COVID-19 cases developed a new onset of diabetes [2]. We want to recommend further details regarding initial screening, the pathogenesis, and supporting examination. We would also like to suggest an alternative differential diagnosis for this patient.

In the case report, the patient was not screened for diabetes on admission. We recommend that all COVID-19 patients be screened for new diabetes, including blood glucose and $\mathrm{HbA} 1 \mathrm{c}$, on admission, during hospitalization, and after recovery. Additionally, other metabolic parameters must be measured to manage the disease better, such as waist circumference. Diabetes is a component of metabolic syndrome closely related to insulin resistance, increasing cardiovascular risk, and mortality.

We are also intrigued by the fact that the patients have positive GAD65 detected. There are reports where the patients with no family history of diabetes developed an insulin-dependent diabetes mellitus following SARS-CoV-2 infection. However, in these cases, no serum autoantibodies were observed [3]. Based on these reports and the fact that the familial history of type 1 diabetes (T1DM) is present, it is necessary to investigate the predisposing genetic factor,

Managed By Massimo Porta.

This comment refers to the article available online at https://doi. org/10.1007/s00592-020-01570-0.

Muhammad Ifham Hanif

muhammadifhamhanif@gmail.com

1 Faculty of Medicine, Universitas Sebelas Maret, Surakarta, Indonesia

2 Faculty of Medicine, Universitas Padjadjaran, Dr. Hasan Sadikin General Hospital, Bandung, Indonesia

3 Department of Internal Medicine, Universitas Sebelas Maret Hospital, Surakarta, Indonesia specifically HLA [4]. Furthermore, we suspected that the patient (in this report) might have previously undetected latent autoimmune diabetes in adults since it shares characteristics with both type 1 (immune-mediated) and type 2 diabetes (adult age at onset).

Moreover, other markers besides GAD65 can be considered, such as the complement systems $(\mathrm{C} 3, \mathrm{C} 4$, factor $\mathrm{D}$, and CD59). Low C-peptide may be associated with not only T1DM but also pancreatitis. We agree with serum lipase being tested because it was more sensitive and specific than amylase. While the patient's CT scan seems to be expected, it may be better to try using USG, MRI, and the final modality using pancreatic biopsy. This case report does not describe the treatment of SARS-CoV-2 infection. There were previous reports of false-positive GAD65 autoantibody test results following IVIG therapy. Hence, there was a possibility of false-positive results.

Viral activation of the renin-angiotensin-aldosterone system and certain medications, mainly glucocorticoids, can induce insulin resistance. Furthermore, this patient had a history of obesity, so we recommend that this patient undergoes the HOMA IR test to determine whether there is insulin resistance or not regarding metformin consideration.

In the case of T1DM, patients should also receive prandial insulin. The latest guideline from the American Diabetes Association recommended that metabolically stable patients receive 0.5 units $/ \mathrm{kg} /$ day of insulin, administered half as prandial insulin and half as basal insulin. However, if the patient's blood sugar control is adequate with basal insulin alone, the diagnosis of T1DM should be re-confirmed. Furthermore, physicians should be aware and continuously monitor blood glucose, thus decreasing the severity and mortality of COVID-19 patients.

The authors questioned the association between diabetes and cytokine storms. SARS-Cov-2 directly attacks beta cells via angiotensin-converting-enzyme-2 (ACE-2) and dipeptidyl-peptidase-4 receptors (DPP-4), hyper-inflammation triggers cytokine storm (TNF- $\alpha$ \& IL-6), which decreases insulin synthesis and systemic insulin resistance, thereby accelerate the onset of diabetes [4]. This hypothesis is 
supported by the findings of the SARS-CoV-2 nucleocapsid protein in the pancreas of postmortem COVID-19 patients. Furthermore, the author's last question about a patient with a massive weight after bariatric surgery seems to develop less severe SARS-CoV-2 infection than subjects suffering from obesity [5].

\section{Declaration}

Conflict of interest All of the authors declare that there were no conflict of interest.

\section{References}

1. Marchand L, Pecquet M, Luyton C (2020) Type 1 diabetes onset triggered by COVID-19. Acta Diabetol 57(10):1265-6
2. Sathish T, Anton MC (2021) Newly diagnosed diabetes in patients with mild to moderate COVID-19. Diabetes Metab Syndr 15(2):569-571

3. Hollstein T, Schulte DM, Schulz J, Glück A, Ziegler AG, Bonifacio E et al (2020) Autoantibody-negative insulin-dependent diabetes mellitus after SARS-CoV-2 infection: a case report. Nat Metab 2(10):1021-1024

4. Li G, Chen Z, Lv Z, Li H, Chang D, Lu J (2021) Diabetes mellitus and COVID-19: associations and possible mechanisms. Int J Endocrinol 2021:1-10

5. Marchesi F, Valente M, Riccò M, Rottoli M, Baldini E, Mecheri F et al (2021) Effects of bariatric surgery on COVID-19: a multicentric study from a high incidence area. Obes Surg 31:2477-2488

Publisher's Note Springer Nature remains neutral with regard to jurisdictional claims in published maps and institutional affiliations. 